\title{
Thigh muscles injuries in professional soccer players: a one year longitudinal study
}

\author{
Angelo Corazza ${ }^{1}$ \\ Davide Orlandi ${ }^{1}$ \\ Amedeo Baldari² \\ Pietro Gatto 3,4 \\ Marco Stellatelli ${ }^{4}$ \\ Claudio Mazzola 5 \\ Roberto Galli6 \\ Stefano Longo 7 \\ Luca Maria Sconfienza7,8 \\ Enzo Silvestri ${ }^{9}$
}

1 Department of Radiology, University of Genova, Italy

2 UC Sampdoria Spa, Genova, Italy

3 Department of Orthopaedics and Traumatology, IRCCS-AOU San Martino IST, Genova, Italy

${ }^{4}$ Genova CFC, Genova, Italy

5 Department of Orthopaedics and Joint Surgery, EO Ospedali Galliera, Genova, Italy

6 Virtus Entella Srl, Chiavari, Genova, Italy

7 Department of Biomedical Sciences for Health, University of Milano, Italy

${ }^{8}$ Department of Radiology, IRCCS Policlinico San Donato, Milano, Italy

9 Department of Radiology and Interventional Ultrasound, Ospedale Evangelico Internazionale, Genova, Italy

Corresponding author:

Angelo Corazza, MD

Department of Radiology

University of Genova

Via L.B.Alberti, 4 - $3^{\text {rd }}$ Floor

16100 Genova, Italy

E-mail: angelcoraz@libero.it

\section{Summary}

Thigh muscles indirect injuries are common finding in soccer and represent a critical challenge for teams medical staffs. Indirect injuries are classified on the basis of their site and their clinical and radiological findings, but the assessment of a precise prognosis remains a crucial point. Both ultrasound (US) and magnetic resonance (MR) represent effective techniques not only to detect indirect injuries but also to accurately determine severity, location, and, consequently, the prognosis. In this setting, our aim is to review imaging findings of professional athletes muscle tears at three time points (3 days, 2 weeks, and 4 weeks after the time of injury) and, further, to investigate the correlation between tears extent and lay-off time of the athletes. Combined US-MR assessment could be helpful in the management of thigh muscles indirect injuries providing accurate information about the site, the extent, and the healing process.

KEY WORDS: muscle injury, thigh, magnetic resonance, ultrasound, soccer.

\section{Introduction}

Thigh muscles injuries represent a common finding in athletes. Recent papers on professional soccer players demonstrated that thigh muscle injuries represent more than $30 \%$ of all injuries and cause the majority of days lost from competition ${ }^{1-5}$. This problem is particularly important in elite clubs where players' performance have also significant financial implications. Hence, if thigh muscles injuries are not critical for the common athlete, at top level an accurate management is mandatory. Usually, history and clinical examination lead to the diagnosis: in most cases muscle injuries occur during activities like sprinting, jumping and kicking, involving muscles that cross two joints (hamstrings, gracilis and rectus femoris), which are made of a high percentage of type 2 fibres, and during eccentric contraction ${ }^{4,6}$. The risk of injury can be increased by cold temperature, inadequate preparation and/or warm-up, excessive fibres stress and previous muscle injuries ${ }^{7,8}$. Recently, team medical staffs increased requests of imaging evaluation, in order to confirm injury, determine its extent and its severity, provide a comprehensive assessment of the muscletendon-bone chain and help the physician to establish a precise prognosis reducing the risk of recurrence. In this setting, magnetic resonance(MR) and ultrasound (US) play a determinant role in the classification of such findings 1,4,5,9-15. Some authors figured out that the size of injury (calculated as the percentage of cross-sectional area of the muscle and/or as the longitudinal length of the tear) and the involvement of the myotendinous junction could provide prognostic information about the return to play 5,9,10,12,15.

With this study we evaluated MR and US in the assessment of both the acute phase and the healing phase of thigh muscles indirect injuries in a cohort of professional soccer players. Further, we investigate the association between the extent of thigh muscles tears and the amount of time lost from competition. 


\section{Methods and Materials}

\section{Study population}

This retrospective study was conducted in accordance with the declaration of Helsinki. Approval of the institutional review board was obtained. Three professional Italian soccer teams (84 males, mean age: $25.4 \pm 5$, range: 16 - 36) were followed at our centre from August 2012 to July 2013, in order to provide radiological evaluation. Full demographics data are reported in Table 1. The athletes used to carry out a weekly activity based on 5 days of 2-3 hours of training, 1 day to dispute the match and 1 day for rest. Team medical staff sent players to our centre for imaging evaluation if clinical findings of thigh muscles injuries were detected. Inclusion criteria for our study were the occurrence of a 'snap' feeling followed or not by loss of function during the sport activity, sensation of a sharp/dull pain at the thigh, stretch-induced or muscle-contraction worsening of pain, presence of visible haematoma and/or palpable defect. Players with history of contusive traumas, long-standing (>20 days) thigh/groin pain, fatigue-related muscular soreness and low-back pain were excluded from our analysis. Our imaging protocol was composed by three combined US-MR examinations: a first evaluation performed within 48-96 hours from injury, a first follow-up between the $13^{\text {th }}$ and the $15^{\text {th }}$ day, and a last follow-up between $30^{\text {th }}$ and $45^{\text {th }}$ day from the injury. In case of negative findings no further follow-up was conducted. Injuries of the same type and occurred at the same site within 2 months after a player's return to play from the former injury were considered as recurrencies ${ }^{5}$. All injured players underwent the same rehabilitative protocol for muscular lesion adopted by the three different club medical staffs, consisting in protection, rest, ice, compression, elevation (PRICE) protocol, physical therapy, isometric to isotonic exercises, strength and functional specific rehabilitation ${ }^{2,16}$. The staff let the athletes return to play when the healing process was considered completed by imaging validation and functional and isokinetic testing on the injured muscle, and the strength imbalances have been corrected.

\section{Magnetic resonance imaging}

MR examinations were conducted at our centre with a high-field magnet (Achieva 1.5 T, Philips, Netherlands) with the athletes lying in a supine position. We performed both longitudinal and axial MR scans of

Table 1. Athletes demographics.

\begin{tabular}{lccc}
\hline Population & n. & $\begin{array}{c}\text { Age (years) } \\
\text { mean } \pm \text { SD;range }\end{array}$ & $\%$ \\
\hline healthy & 57 & $24.6 \pm 5 ; 16-36$ & 67.9 \\
\hline injuried & 27 & $27.1 \pm 4 ; 18-35$ & 32.1 \\
\hline total & 84 & $25.4 \pm 5 ; 16-36$ & 100 \\
\hline
\end{tabular}

pelvis and thigh using a HD 8 channels array. We conducted standardized sequences for each athlete, including a TSE T1-weighted scan (TE: $18 \mathrm{~ms}$; TR: $500 \mathrm{~ms}$ ), a TSE T2-weighted scan without (TE: $70 \mathrm{~ms}$; TR: $3000 \mathrm{~ms}$ ) and/or with fat saturation (TE: $80 \mathrm{~ms}$; TR: $3600 \mathrm{~ms}$ ) oriented on the axial plane, and a STIR(Short Time Inversion Recovery) long-TE scan (TE: 60; TR: 3000; TI: 150) oriented along the sagittal plane if athlete refers tenderness in the anterior or posterior aspect of the thigh, along the coronal plane if tenderness in the medial or lateral aspect of the thigh. A proper field of view was set up for each examination, in relation to the athlete's characteristics. MR images were reviewed by a musculoskeletal radiologist with more than 20 years of experience, who paid particular attention to the possible oedema or structural damage and its precise topography, the presence and entity of possible associated haematomas along fasciae planes and the kind of muscle involved. The extent of the lesion on its major axis was consecutively measured for each injured player with detectable tear on MR. Lesions were classified into four grades following the currently most widely shared MR-based system for indirect muscular injuries: grade 0 , no pathological findings; grade 1, presence of muscle oedema but without tissue damage; grade 2, partial muscle tear and associated fasciae haematoma; grade 3 , a complete muscle tear.

\section{Dynamic high resolution ultrasound}

Ultrasonography was conducted by an experienced musculoskeletal radiologist with high-resolution linear transducers dedicated for the musculoskeletal study (Logiq E9, bandwidth: 15-6 MHz, GE Healthcare, USA; and My Lab 70, bandwidth 13-6 MHz, Esaote, Genoa, Italy); during the scans the athletes laid in a prone position with extended leg for the hamstrings evaluation, in a supine position with extended leg for the quadriceps, in a supine position with abducted leg (flexed and extended) for the adductors. The US examinations consisted in a series of short-axis and long-axis scans of the region of interest of the injured muscle (corresponding to the region of pain), in order to assess the morpho-structural appearance of the muscle fibres (echogenicity, integrity and orientation), the potential tear, the possible presence of intramuscular and/or intra-fasciae haematoma and its extent, the status of the tendons and the possible scar tissue formation. Further, a dynamic evaluation making the patient perform an isometric contraction of the injured muscular group (against appropriate resistance opposed by the operator), was conducted for every athlete; comparative US evaluations were also performed on the contralateral thigh laying in the same previously described position. The extent of the lesion on its major axis was consecutively measured for each injured player with detectable tear on US. Lesions were classified into four grades of severity: grade 0 , no pathological findings; grade 1, altered echotexture at the point of tenderness but no sign of disruption; grade 2, tear and, possibly, associated 
haematoma; grade 3, complete muscle tear. Injuries without evident signs of fibres disruption at static US but with the appearance of subtle structural alteration at the point of tenderness during dynamic scans were classified as grade 1-2 lesions and counted as grade 1 in our study.

\section{Statistical analysis}

We used standard descriptive statistics to report the features of the athletes, mean and SD for the continuous variables and counts and percentages for the categorical variables. Continuous variables as the size of the tears measured on MR and US were compared using the U Mann-Whitney test. We investigated the correlation between the maximal lengths of the tear on its major axis as seen on MR and US and the number of days lost until return to activity with Spearman's nonparametric correlation. The SPSS software (version 17; SPSS Inc., IBM Corporation, Armonk, NY, USA) was used. A $p$ value lesser than 0.05 was considered as significant.

\section{Results}

Twenty-seven indirect thigh muscle injuries were observed, resulting in seventy-one combined US-MR examinations conducted along the study (27 patients at baseline, 25 at 15-days follow-up; 19 at 30-days fol- low-up). We observed two negative findings at the MR baseline evaluations and four negative at the US baseline examinations, six cases of resolution of the lesion at the first follow-up and fifteen resolutions at the last follow-up after 30 days, ten (37\%) of them with the formation of scar tissue.

In respect to lesion location, we found thirteen (48.1\%) hamstrings, six $(22.2 \%)$ adductors and eight (29.6\%) quadriceps muscles injuries in our study, with a rate of recurrence of $14.8 \%$ after $4 \pm 1$ (mean value $\pm S D$ ) weeks (range: $2-6$ ) from the return to play. Myotendinous junction resulted the most involved site with 18 (11 grade 2; 7 grade 1) out of 25 $(64 \%)$ injuries occurred in such area. The majority of the lesions ( $n=15,55.5 \%)$ were grade 2 tears, grade 1 lesions have been found in ten cases (37\%). MR tear detection rate was higher than US (25/27 vs 23/27).

Considering the 15 grade 2 lesions measured at baseline, the maximal length of the tears resulted larger at MR evaluation than that detected at US $(29 \mathrm{~mm} \pm$ 18 (mean value $\pm \mathrm{SD}$ ); $27 \mathrm{~mm} \pm 17$ respectively). Full data are reported in Table 2. No differences between tear size measured at MR and US were observed at follow-up ( $p=0.678)$. Overall, athletes in our study had to lay-off from competition for a mean time of 20 days \pm 9 , range: $6-46$ days; for injured players with grade 2 tears the mean lay-off time was 25 days \pm 9 , range: 13-46 days. High correlation between fibres tear size and athlete's lay-off time was found $[r=0.88(p<0.001)$, for MR measurements; $r=0.86(p<0.001)$, for US measurements], (Fig. 1).

Table 2. Pathological findings.

\begin{tabular}{|c|c|c|c|c|}
\hline & grade 0 & grade 1 & grade 2 & Total \\
\hline injuries & $2(7.4 \%)$ & $10(37 \%)$ & $15(55.5 \%)$ & $27(100 \%)$ \\
\hline hamstrings & 1 & 5 & 7 & $13(48.1 \%)$ \\
\hline adductors & 0 & 2 & 4 & $6(22.2 \%)$ \\
\hline quadriceps & 1 & 3 & 4 & $8(29.6 \%)$ \\
\hline recurrence & 1 & & & \\
\hline - hamstrings & & 0 & 1 & $4(14.8 \%)$ \\
\hline - adductors & & 1 & 0 & \\
\hline - quadriceps & & 0 & 2 & \\
\hline $\begin{array}{l}\text { tear size on } M R \\
- \text { mean } \pm S D(\mathrm{~mm}) \\
\text { - range (days) }\end{array}$ & I & I & $\begin{array}{c}29 \pm 18 \\
8-63\end{array}$ & I \\
\hline $\begin{array}{l}\text { tear size on US } \\
\text { - mean } \pm S D(\mathrm{~mm}) \\
\text { - range (days) }\end{array}$ & 1 & I & $\begin{array}{c}27 \pm 17 \\
7-58\end{array}$ & I \\
\hline $\begin{array}{l}\text { lay-off time } \\
\text { (days; mean } \pm S D \text { ) }\end{array}$ & $5 \pm 1$ & $13 \pm 5$ & $25 \pm 9$ & $20 \pm 9$ \\
\hline
\end{tabular}




\section{Discussion}

When a muscular injury is suspected, it is very important to start with a precise anamnesis followed by a careful clinical examination of the muscles and an accurate imaging evaluation; hence, it is necessary to have a shared definition and a clear characterization of each type of muscle injury between operators of different fields. Previous published muscle injuries classification systems have been based on the severity, site and/or etiopathology of the lesions. Takebayashy et al. ${ }^{17}$ in 1995 and Peetrons ${ }^{18}$ in 2002 proposed ultrasound-based grading systems that classifies muscle injuries into three grades basing on the severity; Stoller ${ }^{19}$ in 2007 proposed a 3 grades classification based on MR findings; more recently Chan et al. ${ }^{15}$ presented a new classification based on the site of the lesion. In our examinations we classified MR findings into four grades of severity; we found two cases with negative findings (grade 0); ten injured muscles with presence of intramuscular oedema without fibres damage (grade 1), fifteen cases with detection of tear and fibres distortion with possible associated fasciae haematoma (grade 2), (Fig. 2). No complete ruptures of the muscle (grade 3 ), were found. In US evaluations, we found four negative cases (grade 0); ten findings such as focal echogenicity alterations and focal fi-

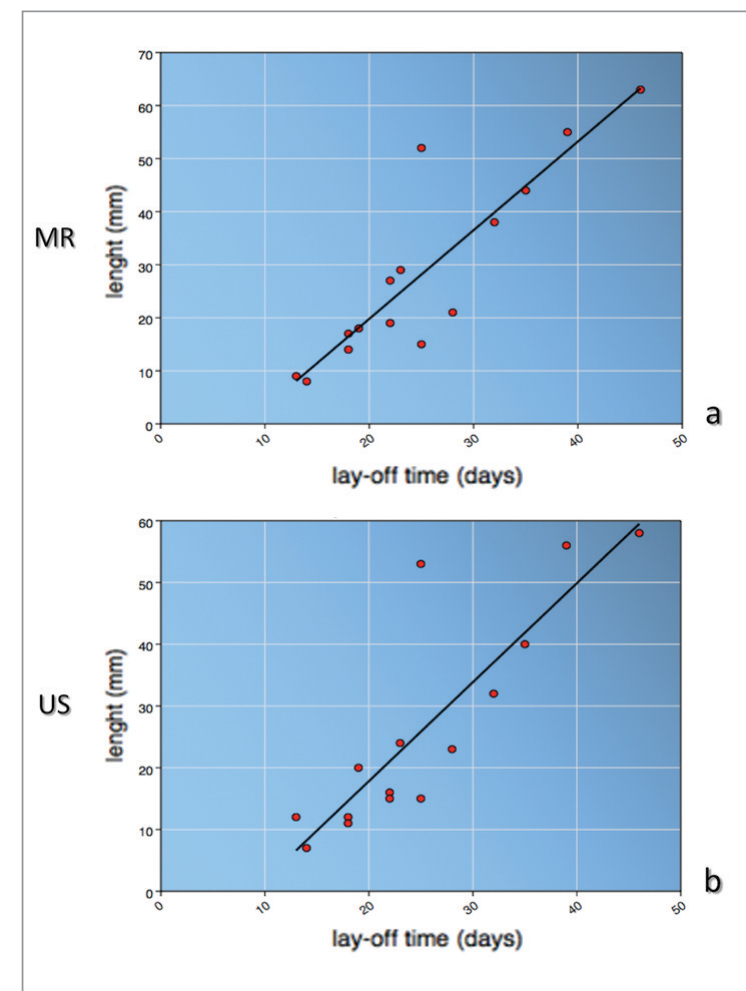

Figure 1. Scatterplot and line of best fit which illustrate the correlation between the length of tear as seen on MR (a) and US (b) and the number of days until return to play. Spearman's rank order correlation coefficient $(r)$ was 0.88 for MR measurements and 0.86 for US measurements. bres orientation abnormalities at the point of tenderness, addressed as grade 1 injuries; fifteen cases with fibres distortion, tear and haemorrhage at the point of damage and/or along fasciae planes, classified as grade 2 lesions. Moreover, we experienced that, at the US examination, five minor injuries classified as grade 1, revealed themselves only with dynamic evaluation: in our routine we use to characterize them as intermediate grade 1-2 tears but in this investigation, they were conventionally addressed as grade 1 tears, in order to have more reproducible results.

Regarding to the diagnostic features of MR and US, in our series MR found alterations in two patients where US did not reveal any pathological finding. In these two cases (one player who reported pain in the antero-lateral aspect of the thigh with MR finding of subtle area of fasciae oedema in the deep medial portion of the vastus lateralis, and another athlete with pain in the posterior middle thigh and showing on MR a subcentimetric area of altered signal at the short head of the biceps femoris) MR and US were not in agreement, reflecting the limit of US examinations in the assessment of minor, deep located lesions $8,9,18$. On the other side, it could be explained by the greater sensi-

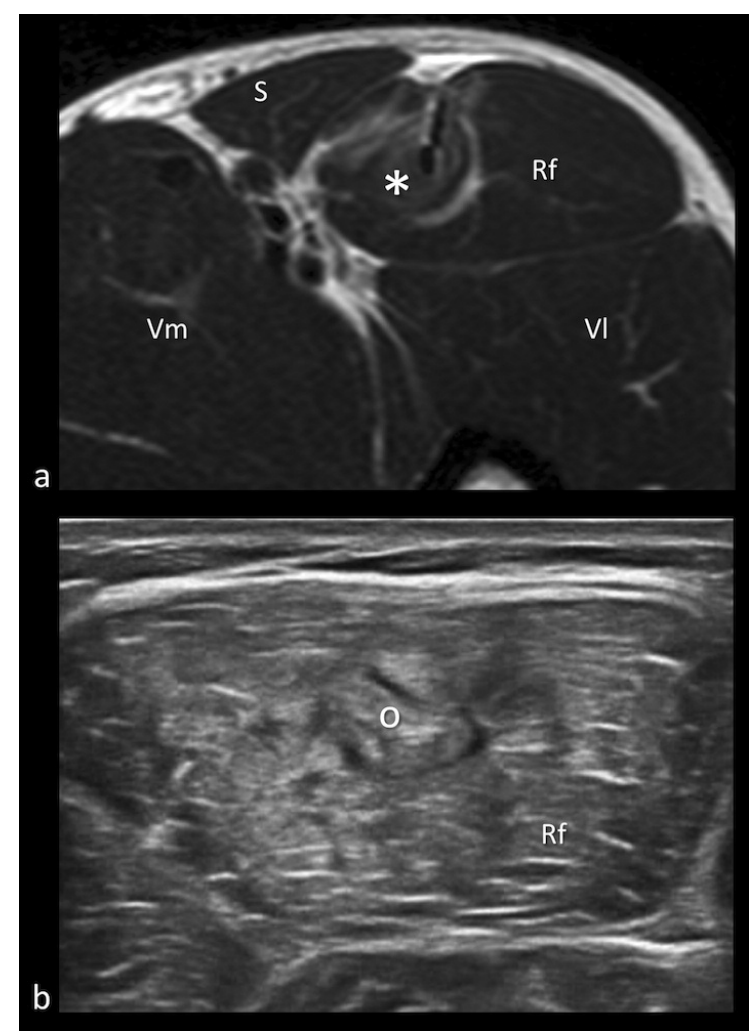

Figure 2. Rectus Femoris grade 2 injury. a) axial T2weighted MR scan at the level of the proximal third of the thigh, showing a tear in correnspondence of the left rectus femoris aponeurosis with oedema and haemorrhage of the myo-tendinous junction (asterisk). b) dynamic US axial scan during muscle contraction revealing the fibres damage (circle). Rf: rectus femoris; S: sartorius; VI: vastus lateralis; Vm: vastus medialis. 
tivity of MR for the detection of intramuscular oedema ${ }^{9}$ on fluid-sensitive fat-saturated sequences. This finding results particularly evident in minor lesions, where the ability to diagnose them effectively is strictly related to the sole presence of oedema in the injured mus$\mathrm{cle}^{1,8}$. In every other injuries MR and US showed no significant difference in the detection of the abnormalities. Both imaging procedures resulted effective diagnostic techniques for the detection of almost all of the injuries, resulting in agreement for 23 out of 25 pathological findings.

An additional consideration was made by Ekstrand et al. ${ }^{5}$, who found that about $70 \%$ of hamstring injuries seen in professional soccer showed no signs of fibres disruption on MR. In our study we found 12 injuries (two grade 0 and ten grade 1 lesions) with no sign of fibres structural damage on MR, corresponding to $44.4 \%$ of all injuries; this finding could be due to a selection bias given by the clinical examinations performed by team medical staff prior to send athletes to imaging evaluation. Counting that five of the grade 1 were actually grade 1-2 lesions, without them the percentage of injuries with no signs of disruption at any imaging examination would be $26 \%$ indicating the high diagnostic efficacy reached by the combination of dynamic high-resolution US, high-field MR and experienced radiologist.

Hamstrings resulted the most involved compartment of the thigh reflecting the higher prevalence of hamstrings and rectus femoris injuries in professional athletes $3,4,8,13,20-22$.

Myo-tendinous junction was the most involved site reflecting the fact that it represents the weakest point of the muscle-tendon-bone chain, confirming the findings of recent papers $4,5,8-10,13,15,20$. Connell et al. ${ }^{9}$ reported no significant difference between lay-off times deriving from myo-tendinous junction versus myo-fasciae injuries. Our results agree with his data and, further, add that injuries involving the myo-tendinous junction had longer time to recovery as compared with muscle belly injuries.

Some recent studies ${ }^{1,3,4,5,9,10,23}$ reported the significant correlation existing between the lay-off time and the cranio-caudal length and/or cross-sectional area of the tear or of the whole altered tissue region. According to them, our study reports sharp correlation between the extent of the tear and the lay-off time of the athlete, arguing that it could be a useful predictor for the recovery time: grade 1 injuries involving a little amount of myofibrils only required less than 15 days to recovery; grade 2 more severe injuries with extended fascicular damage and intra/extra muscular haematoma required a longer period to heal. In three cases the healing process took more than $4 / 5$ weeks to complete and, at the last follow-up, imaging showed persistent pathological signs: this could be explained because of the specific location of those injuries: one of them was located at the rectus femoris proximal aponeurosis and the other two lesions were sited at the proximal teno-muscular junction of the common semitendinosus-biceps femoris tendon, suggesting that a little quote of tendon fibres had been actually damaged prolonging the regenerative process, as reported also on some other papers ${ }^{1,9,15}$.

Since our study included injuries to all muscles of the thigh and not only to the hamstrings, we found some technical problems to make measurements of craniocaudal length/cross-sectional area; this was solved performing measurements on the major axis of tears in order to give a more adequate evaluation of the lesions. MR and US did not show significant difference in the measurements of the tear extent at follow-up; in some cases MR could overestimate the extent of the altered tissue ${ }^{8,9}$, particularly at the early diagnostic evaluation.

As reported by other authors ${ }^{7,9}$, in our study athletes were symptoms-free within relatively brief period, although the substanding regenerative process and the relative rehabilitation period was not completed. This finding expresses the main challenge for professional soccer teams, which is to conjugate the demand of an early return to competition with the risk of recurrence and, consequently, a more prolonged period of convalescence. Our study is aligned with literature da$\mathrm{ta}^{1,3,4,23}$ and reports four (14.8\%) cases of recurrence after 2 - 6 weeks, occurred at the same muscle interested by the first injury.

These result are also comparable with those reported by Malliaropoulos et al. ${ }^{23}$ who observed a $13.9 \%$ recurrence rate after a clinically-based thigh muscle injuries management. Although a direct comparison could not be performed because of the different injury grading scale and the different muscle groups included in our study, we observed a $8.3 \%$ hamstrings injury recurrence rate. Obviously, the small sample size affects the power of this observation, but it may reflect the efficacy of a combined imaging-based follow-up. Moreover, we strongly agree with Malliaropulos et al. ${ }^{24}$ about the need to identify any reversible risk factors that may further increase the risk of re-injury of athletes with a previous muscle tear.

In this setting, scar formation and consequent higher risk of recurrence ${ }^{3,6}$ represent one of the most difficult challenges for the appropriate rehabilitation of the athlete. Thus, the use of sonoelastography, recently proposed as an integrative tool to standard US in musculoskeletal pathology $25-27$, may be potentially useful, in particular to evaluate the intrinsic structural features of the scar (elasticity, stiffness) and also to assess the entire healing process of injured muscle fibres. Further investigations should be aimed to demonstrate whether sonoelastography can depict intrinsic features of muscular injuries and scar tissue, and to understand whether such information may potentially modify the rehabilitative approach to those lesions.

Our study has some limitations: first of all the number of athletes followed with our imaging protocol was quite limited and they were also represented by elite players only, not reflecting the features of a more general population. Further, the intrinsic heterogeneity of thigh muscles (unlike many other studies which focused on hamstrings $\left.{ }^{1,5,9-12,14,23}\right)$, the different location and severity of injuries may constitute a limitation. In contrast, the homogeneity of our study population, 
made by male professional soccer players of 16 to 36 year-old, followed with standard protocol along the entire healing process, could be seen also as a strength of our study. The high performance of US reported could be reached because of availability of high-level technology and the experience of the musculoskeletal radiologist who performed all the US examinations as the only observer; we can not be sure that comparable results can be obtained in every day clinical routine.

Summarizing, combined dynamic US and MR imaging allow to cover all diagnostic steps that follows through the professional athlete's injury and rehabilitation, helping clinicians to precisely characterize thigh injuries and more accurately estimate the time required before an athlete can return to competition as well as the related risk of recurrent injury. We can speculate that in clinical routine MR imaging could be considered as the first choice technique for the detection of thigh muscle injuries, in particular for minor lesions where the damage occurring to the myofibrils may not be visualized with US alone whereas the increase of MR intensity helps to make the correct diagnosis. At the consequent followups, even if MR and US got comparable results in the evaluation of the recovery during rehabilitation, US may be preferred, for the availability, the cost-benefit profile and the dynamic feature best fitting for the assessment of the healing process both on the anatomical and on the functional aspect.

The current study highlights the effectiveness of combined US-MR imaging in the evaluation of thigh muscular injuries, which plays a determinant role in the management of elite soccer players. In our experience, it is very important to use high-quality imaging technology and to collaborate with the team medical staff in order to accurately plan and guide athlete rehabilitation obtaining the shortest return-to-play time with the minimum risk for the athlete.

\section{Acknowledgments}

The authors wish to thank the clubs, medical staffs and players participating the study.

\section{References}

1. Rubin DA. Imaging Diagnosis and Prognostication of Hamstring Injuries. AJR Am J Roentgenol 2012; 199(3):525-533.

2. Clanton TO, Coupe KJ. Hamstring strains in athletes: diagnosis and treatment. J Am Acad Orthop Surg 1998; 6(4):237248.

3. Ekstrand J, Hagglund M, Walden M. Epidemiology of muscle injuries in professional football (soccer). Am J Sports Med 2011; 39(6):1226-1232.

4. Mueller-Wohlfahrt HW, Haensel L, Mithoefer K, et al. Terminology and classification of muscle injuries in sport: a consensus statement. Br J Sports Med 2013; 47(6):342-350.

5. Ekstrand J, Healy JC, Waldén M, Lee JC, English B, Hägglund $\mathrm{M}$. Hamstring muscle injuries in professional football: the correlation of MRI findings with return to play. Br J Sports Med 2012; 46(2):112-117.

6. Jarvinen TA, Jarvinen TL, Kaariainen M, et al. Muscle injuries: biology and treatment. Am J Sports Med 2005; 33(5):745764.

7. Garrett WE Jr. Muscle strain injuries. Am J Sports Med 1996; 24(6):S2-8.

8. Megliola A, Eutropi F, Scorzelli A, et al. Ultrasound and magnetic resonance imaging in sports-related muscle injuries. Radiol Med 2006; 111(6):836-845.

9. Connell DA, Schneider-Kolsky ME, Hoving JL, et al. Longitudinal Study Comparing Sonographic and MRI Assessments of Acute and Healing Hamstring Injuries. AJR Am J Roentgenol 2004; 183(4):975-984.

10. Slavotinek JP, Verrall GM, Fon GT. Hamstring Injury in Athletes: Using MR Imaging Measurements to Compare Extent of Muscle Injury with Amount of Time Lost from Competition. AJR Am J Roentgenol 2002; 179(6):1621-1628.

11. Malliaropoulos NG. Non contact hamstring injuries in sports. Muscles Ligaments Tendons J 2013; 2(4):309-311.

12. Kerkhoffs GM, van Es N, Wieldraaijer T, Sierevelt IN, Ekstrand $\mathrm{J}$, van Dijk CN. Diagnosis and prognosis of acute hamstring injuries in athletes. Knee Surg Sports Traumatol Arthrosc 2013; 21(2):500-509.

13. Armfield DR, Kim DH, Towers JD, Bradley JP, Robertson DD. Sports-related muscle injury in the lower extremity. Clin Sports Med 2006; 25(4):803-842.

14. Askling CM, Tengvar M, Saartok T, Thorstensson A. Acute first-time hamstring strains during high-speed running: a longitudinal study including clinical and magnetic resonance imaging findings. Am J Sports Med 2007; 35(2):197-206.

15. Chan O, Del Buono A, Best TM, Maffulli N. Acute muscle strain injuries: a proposed new classification system. Knee Surg Sports Traumatol Arthrosc 2012; 20(11):2356-2362.

16. Heiderscheit BC, Sherry MA, Silder A, Chumanov ES, Thelen DG. Hamstring strain injuries: recommendations for diagnosis, rehabilitation, and injury prevention. J Orthop Sports Phys Ther 2010; 40(2):67-81.

17. Takebayashi S, Takasawa H, Banzai $Y$, et al. Sonographic findings in muscle straininjury: clinical and MR imaging correlation. J Ultrasound Med 1995; 14(12):899-905.

18. Peetrons P. Ultrasound of muscle. Eur Radiol 2002; 12(1):35-43.

19. Stoller DW. MRI in orthopaedics and sports medicine 3rd edn. Philadelphia; Wolters Kluwer/Lippincott 2007.

20. Lee JC, Mitchell AW, Healy JC. Imaging of muscle injury in the elite athlete. Br J Radiol 2012; 85(1016):1173-1185.

21. Volpi P, Melegati G, Tornese D, et al. Muscle strains in soccer: a five-year survey of an Italian major league team. Knee Surg Sports Traumatol Arthrosc 2004; 12(5):482-485.

22. Armfield DR, Hyun-Min Kim D, Towers JD, Bradley JP, Robertson DD. Sports-Related Muscle Injury in the Lower Extremity. Clin Sports Med 2006; 25(4):803-842.

23. Malliaropoulos N, Papacostas E, Kiritsi O, Papalada A, Gougoulias N, Maffulli N. Posterior thigh muscle injuries in elite track and field athletes. Am J Sports Med 2010; 38(9):1813-1819.

24. Malliaropoulos N, Isinkaye T, Tsitas K, Maffulli N. Reinjury after acute posterior thigh muscle injuries in elite track and field athletes. Am J Sports Med 2011; 39(2):304-310.

25. Sconfienza LM, Silvestri E, Cimmino MA. Sonoelastography in the evaluation of painful Achilles tendon in amateur athletes. Clin Exp Rheumatol 2010; 28(3):373-378.

26. Sconfienza LM, Silvestri E, Orlandi D, et al. Real-time sonoelastography of the plantar fascia: comparison between patients with plantar fasciitis and healthy control subjects. Radiology 2013; 267(1):195-200.

27. Cosgrove D, Piscaglia F, Bamber J, et al. EFSUMB guidelines and recommendations on the clinical use of ultrasound elastography. Part 2: Clinical applications. Ultraschall Med 2013; 34(3):238-253. 